\title{
A Case of Neurosyphilis Presenting With Tongue Tremor
}

\author{
Adriana Moro ${ }^{\mathrm{a}}$, Mariana Moscovich ${ }^{\mathrm{a}}$, Francis Paciornik Zorzetto ${ }^{\mathrm{a}}$, \\ Renato Puppi Munhoz ${ }^{\mathrm{b}}$, Helio A. Ghizoni Teive ${ }^{\mathrm{a}, \mathrm{c}}$
}

\begin{abstract}
There are three common forms of neurosyphilis: asymptomatic, meningeal and vascular. Another rarer presentation is the parenchymatous form, which includes general paresis and tabes dorsalis. Although ataxia is present in tabes dorsalis, other movement disorders represent rare manifestations of neurosyphilis. A 32-year-old man presented with dementia and tongue tremor, in addition to generalized bradykinesia. Diagnosis of neurosyphilis was done and patient was treated with penicillin $\mathrm{G}$ cristalin. Six months later, neurological examination showed improvement of the tongue tremor. This case report highlights a rare cause of movement disorder, with a peculiar sign of neurosyphilis.
\end{abstract}

Keywords: Neurosyphilis; Tongue tremor; Dementia; Movement disorder

\section{Introduction}

Considering the current trend towards an increasing rate of neurosyphilis in several parts of the world, it is essential for the general practitioner to be able to recognize its different clinical presentations, as effective forms of treatment are widely available [1-3]. The diagnosis of neurosyphilis is defined by the presence of positive serum venereal disease research laboratory (VDRL) or fluorescent treponemal antibody - absorption (FTA-Abs) tests, and abnormal cere-

Manuscript accepted for publication August 13, 2014

\footnotetext{
${ }^{a}$ Movement Disorders Unit, Neurology Service, Hospital de Clinicas of Federal University of Parana, Curitiba, Parana, Brazil

${ }^{\mathrm{b}}$ University of Toronto, Department of Medicine, Morton and Gloria Shulman Movement Disorders Center, Toronto Western Hospital, Toronto, ON, Canada

${ }^{\mathrm{c}}$ Corresponding Author: Helio A. Ghizoni Teive, Neurology Service, Hospital de Clinicas, Federal University of Parana, Rua General Carneiro 1103/102, Centro, Curitiba, Parana, Brazil.

Email: hagteive@mps.com.br
}

doi: http://dx.doi.org/10.14740/jnr290w brospinal fluid (CSF) analysis (VDRL and pleocytosis). The three common forms of neurosyphilis are asymptomatic, meningeal and vascular. Another rarer presentation is the parenchymatous form, which includes general paresis (dementia paralytica) and tabes dorsalis $[1,2]$.

Tabes dorsalis, also known as progressive locomotor ataxia, is characterized by progressive ataxia, loss of proprioception, absent deep tendon reflexes, Argyll-Robertson pupils and neuralgic paroxysmal pain. Movement disorders, other than ataxia, represent rare manifestations of neurosyphilis $[1,2]$.

Here we present a peculiar case of neurosyphilis presenting with tongue tremor and dementia.

\section{Case Report}

A 32-year-old man presented with a 2-year history of progressive aggressiveness, visual and auditory hallucinations, persecutory delusions, anhedonia, apathy, and multi-domain cognitive deficits that included memory, writing, reading and calculation difficulties. On neurological examination, mini-mental status examination (MMSE) score was 17. Argyll-Robertson pupils were absent. A coarse rest and action tongue tremor were observed, associated with generalized bradykinesia. Other features such as rigidity, postural instability or tremor in other body parts were absent. Routine blood tests were normal, except for a reactive VDRL with a 1:16 titer and a positive FTA-Abs test. Brain CT and MRI scans showed signs of generalized atrophy. CSF analysis showed the presence of 69 leukocytes ( $90 \%$ lymphocytes), elevated protein $(162 \mathrm{mg} / \mathrm{dL})$, low glucose $(47 \mathrm{mg} / \mathrm{dL})$ and reactive VDRL with a 1:64 titer. The patient was treated with penicillin $\mathrm{G}$ cristalin 24 million units, intravenously for 14 days, with moderate improvement of cognitive functions. After a 6-month follow-up, neurological examination showed improvement of the tongue tremor. CSF analysis showed 11 leukocytes (95\% lymphocytes), protein $42 \mathrm{mg} /$ $\mathrm{dL}$, glucose $58 \mathrm{mg} / \mathrm{dL}$ and VDRL 1:2. Another CSF analysis performed after 18 months showed four leukocytes, glucose $64 \mathrm{mg} / \mathrm{dL}$ and protein $33 \mathrm{mg} / \mathrm{dL}$, with VRDL 1:2. Cognitive function was also markedly improved with MMSE of 23. 
Table 1. Tongue Movement Disorders in Acquired Neurosyphilis

Tongue movement disorder

1. Trombone tremor of the tongue

2. Trombone tongue

3. Stereotypical dyskinesia

4. Orofacial chorea

5. Candy sign

6. Coarse tongue tremor
Reference

References 7

References 7,8 and 10

Reference 7

Reference 7

Reference 7

Present case

\section{Discussion}

Classically, the clinical syndromes of neurosyphilis that are more widely recognized are general paresis, also known as dementia paralytica, or Lissauer form, and tabes dorsalis or progressive locomotor ataxia $[1,2]$. Movement disorders associated with neurosyphilis are typically sensory ataxia, parkinsonism, myoclonus, chorea and dystonia [3]. Other very rare movement disorder syndromes include progressive supranuclear palsy, corticobasal, and cerebellar ataxia/myoclonus syndromes $[3,4]$. Tong et al [5] evaluated the spectrum of movement disorders secondary to neurosyphilis. The authors studied seven of the 169 neurosyphilis (with syphilitic meningitis, meningovascular neurosyphilis, general paresis and tabes dorsalis) patients presenting with movement disorders, including four patients with parkinsonism, one each with laryngeal dystonia, corticobasal syndrome and sensory ataxia.

General paresis is characterized by progressive cognitive impairment (evolving to severe dementia), with pyramidal signs, Argyll-Robertson pupils, and psychiatric symptoms (emotional lability, paranoia, delusions of grandeur, hallucinations) and bradykinesia $[1,2,6]$. The case presented here is characteristic of general paresis, a parenchymatous form of neurosyphilis, associated with tongue tremor. In this rare clinical syndrome of neurosyphilis, the more commonly reported movement disorder is a very characteristic coarse tongue tremor, occasionally associated with facial and upper limbs tremor [2, 7, 8]. Silverdale et al [9] studied the spectrum of orolingual tremor and proposed a new syndrome classification. In this review the authors defined other secondary causes of orolingual tremors, including neurosyphilis.

Recently Martinelli et al [7] described a very unique orofacial dyskinesia related to neurosyphilis. This kind of dyskinesia, involving the lips and lower facial muscles, was named the "candy sign" and was considered pathognomonic of neurosyphilis [7]. There are other descriptions of tongue movement disorders in neurosyphilis, such as orofacial chorea, stereotypical dyskinesia, and trombone tremor of the tongue or trombone tongue $[6-8,10]$ (Table 1).

\section{Conflict of Interest}

The authors have nothing to disclose.

\section{Fund Support}

This article is not funded by any source.

\section{References}

1. Marra CM. Update on neurosyphilis. Curr Infect Dis Rep. 2009;11(2):127-134.

2. Ghanem KG. REVIEW: Neurosyphilis: A historical perspective and review. CNS Neurosci Ther. 2010;16(5):e157-168.

3. Shah BB, Lang AE. Acquired neurosyphilis presenting as movement disorders. Mov Disord. 2012;27(6):690695.

4. Shah BB, Lang AE. A case of neurosyphilis presenting with myoclonus, cerebellar ataxia, and speech disturbance. Mov Disord. 2012;27(6):794.

5. Tong ML, Lin LR, Zhang HL, Huang SJ, Liu GL, Zheng $\mathrm{WH}, \mathrm{Su} \mathrm{YH}$, et al. Spectrum and characterization of movement disorders secondary to neurosyphilis. Parkin- 
sonism Relat Disord. 2013;19(4):441-445.

6. Howard R, Manji H. Infection of the nervous system. In: Clarke C, Howard R, Rossor M, Shorvon S, eds. Neurology. A Queen Square Textbook. Oxford: WileyBlackwell; 2009:289-335.

7. Martinelli P, Rizzo G, Scaglione C, Capellari S. Neurosyphilis orofacial dyskinesia: the candy sign. Mov Disord. 2013;28(2):246-247.

8. Donaldson IM, Marsden CD, Schneider SA, Bathia KP.
Isolated site-specific or task-specific tremors. In: Donaldson IM, Marsden CD, Schneider SA, Bathia KP, eds. Marsden's book of movement disorders. Oxford: Oxford University Press; 2012:691-707.

9. Silverdale MA, Schneider SA, Bhatia KP, Lang AE. The spectrum of orolingual tremor--a proposed classification system. Mov Disord. 2008;23(2):159-167.

10. Worster-Drough C. Neurosyphilis. London: John Bale, Sons \& Staples; 1940. 